\title{
Progress on ELI-Beamlines 10 PW Laser System
}

\author{
Gilles CHÉRIAUX, ${ }^{1}$ Roman ANTIPENKOV, ${ }^{2}$ Frantisek BATYSTA, ${ }^{2}$ Ted BORGER, ${ }^{1}$ \\ Gavin FRIEDMAN,,$^{1}$ Jonathan Tyler GREENE, ${ }^{2}$ Doug HAMMOND, ${ }^{1}$ Jimmy HEISLER, ${ }^{1}$ \\ Axel JOCHMANN, ${ }^{1}$ Matt KEPLER, ${ }^{1}$ April KISSINGER, ${ }^{1}$ Daniel KRAMER, ${ }^{2}$ Jean-Claude LAGRON, ${ }^{2}$ \\ Alexander MEADOWS, ${ }^{2}$ Bedrich RUS,${ }^{2}$ Pavel TROJEK, ${ }^{2}$ Stepan VYHLÍDKA, ${ }^{2}$ \\ Erhard GAUL, ${ }^{1}$ and Todd. DITMIRE ${ }^{1}$ \\ ${ }^{1}$ National Energetics, 4616 W. Howard Lane, Austin, TX 78728, USA \\ ${ }^{2}$ Extreme Light Infrastructure-Beamlines, Institute of Physics, FZU AS CR, v.v.i., Za Radnicí 835, 25241 Dolní Břežany, Czech Republic
}

(Received December 17, 2017)

\begin{abstract}
The $10 \mathrm{PW}$ laser system under construction for ELI-Beamlines will exhibit output pulses delivered every minute with an energy of $1.5 \mathrm{~kJ}$ within $150 \mathrm{fs}$ duration. The laser is a hybrid OPCPA/glass laser system. Here we present results on the high repetition rate front-end and on the first power amplifier.
\end{abstract}

Key Words: Ultrafast laser, Laser amplification, Optical Parametric Chirped Pulse Amplification (OPCPA), Glass amplifiers, High power laser

\section{Introduction}

Over the past decades, high peak power laser systems have been paving the way for new physics experiments and very promising results in generating secondary sources of either radiation as X-Rays or V-UV or particles as electrons or protons have been obtained. The state of the art of high peak intensity lasers is currently above $4 \mathrm{PW}$, which was achieved with titanium doped sapphire crystal laser gain media. ${ }^{1)}$ Many programs are aiming for even higher peak powers up to $10 \mathrm{PW}$. The technical solutions for obtaining such peak powers is through short pulses amplification via Chirped Pulse Amplification (CPA) scheme. The most commonly used laser material for these systems is the titanium doped sapphire whose main advantages are the very broad spectral bandwidth and the saturation fluence level. ${ }^{2,3)} \mathrm{An}$ other possible solution is to use Optical Parametric Chirped Pulse Amplification (OPCPA) in non-linear crystals such as $\mathrm{BBO}, \mathrm{LBO}$ and $\mathrm{KDP}^{4-6)}$ This scheme is particularly interesting in regards of the spectral bandwidth. ${ }^{7)}$ On the other hand, Nd:glass based laser system have demonstrated high energy possibility ${ }^{8,9)}$ and also the potential for short pulse duration. ${ }^{10^{-13)}}$ Nevertheless, all these glass-based systems only have a repetition rate of few shots a day; they are limited in repetition rate by the thermal load in the glass slabs due to flash lamp energy deposition because they rely on passive cooling of the laser medium.

The requirements for the ELI-Beamlines $10 \mathrm{PW}$ laser are 1500 Joules in $150 \mathrm{fs}$ at 1 shot a minute. To achieve these high requirements, the 10PW laser has been designed on a hybrid scheme of OPCPA and actively cooled glass amplification. The system is described on Fig. 1.

The Front-End (FE) is based on OPCPA for temporal contrast and spectral bandwidth management. Two Power Amplifiers (PA) based on liquid cooled split-disk and mixed glass technology are used for thermal and spectral management, and efficient energy amplification. The pulses are then transported through a Compressor Imaging System (CIS) to the optical compressor.

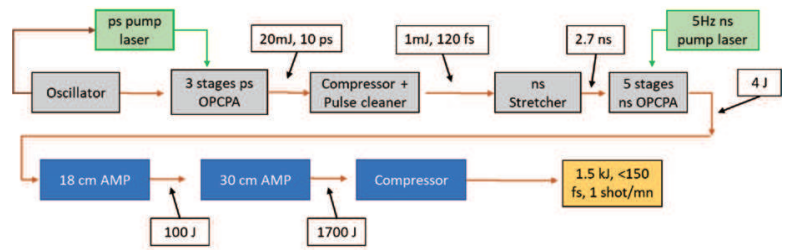

Fig. 1 Layout of $10 \mathrm{PW}$ laser system. Gray boxes indicate subsystems in the laser front end, green indicate pump lasers for front end amplification, and blue indicate high energy, glass-based amplifiers and compressor. Energies and pulse durations are indicated between stages. It should be emphasized that the $100 \mathrm{~J}$ and $1700 \mathrm{~J}$ energies shown are predicted values and have not yet been fully implemented.

\section{Front-end}

The Front-End (FE) must fulfill several requirements for final pulse characteristics: a large spectral bandwidth centered at $1060 \mathrm{~nm}$ compatible with a $130 \mathrm{fs}$ Fourier Transformed Limited (FTL) duration, > $200 \mathrm{ps} / \mathrm{nm}$ stretching factor for limited B-integral during amplification, $>10^{12}$ temporal contrast compatible with $10^{22} \mathrm{~W} / \mathrm{cm}^{2}$ focused intensity, spectral and spatial shaping possibilities, and synchronization (jitter $<100 \mathrm{fs}$ ) of the amplified pulse with an external masterclock provided by the facility. To achieve these high-end performances the FE is base on OPCPA of the output of a modelocked femtosecond oscillator. The FE can be divided into five sections as shown in Fig. 1 a modelocked fiber oscillator, a picosecond OPCPA section, a temporal management section (pulse cleaner and stretcher) and a final nanosecond OPCPA bringing the pulse energy to more than 4 Joules in a square beam at $5 \mathrm{~Hz}$ repetition rate. 
A fiber oscillator with broad output spectrum (50 fs FTL) locked to an external reference clock signal provides the seed for both ps OPCPA and ps pump laser. Three stages BBO picosecond OPCPA produce pulse energy up to $20 \mathrm{~mJ}$ at $5 \mathrm{~Hz}$. The pulses are compressed to $120 \mathrm{fs}$ before entering the pulse cleaner based on a low gain degenerated OPA scheme. ${ }^{14,15)}$ In the pulse cleaner, the compressed picosecond OPCPA pulse is split in two; $90 \%$ is converted to the second harmonic that will be the pump pulse in the parametric process and the remaining $10 \%$ will be the signal. With appropriate balancing between the different waves, an idler with an energy of $2 \mathrm{~mJ}$ at the same wavelength as the signal is generated. Thanks to this three waves process, the output pulse goes as $I_{\text {signal }}(t)^{3}$. Figure 2 shows a third order correlation measurement of the pulse after the pulse cleaner. The actual noise level of the third order correlator is $7 \times 10^{-9}$.

The solid line curve is the experimental one and the dashed curve is the calculated $I_{\text {signal }}(t)^{3}$.

The temporally filtered pulse exhibits a very low level of pedestal limited on this measurement by the noise level of the third order correlator. The pulse is very sharp and fits with the calculated one.

The cleaned pulses are then directed into an Öffner-type stretcher ${ }^{16}$ under vacuum. The stretcher uses a single 1136 lines $/ \mathrm{mm}$ dielectric coated grating used at Littrow incidence angle. A four passes configuration is used to obtain a stretching ratio of $214 \mathrm{ps} / \mathrm{nm}$. The pulse is then transported into the nanosecond OPCPA section which consists of five stages pumped by Nd:YAG lasers (NL940 from EKSPLA). The pump pulses are temporally tailored for optimum shaping of the output amplified spectrum. Indeed, spectral gain narrowing in the followings two glass PAs would lead to a narrow spectrum for the final 1700 Joules pulses if the input spectrum was not strongly shaped. Simulations have been carried out to find the optimal temporal shapes of the five different pump lasers considering the input stretched pulse spectrum and the gain bandwidth of the subsequent glass amplifiers. Applying the result to the pump lasers for five stages, amplification has been obtained up to $4 \mathrm{~J}$ at $5 \mathrm{~Hz}$. Figure 3 shows the amplified spectrum.

The beam quality throughout the laser chain is also a critical aspect. The beam is spatially shaped from circular Gauss-

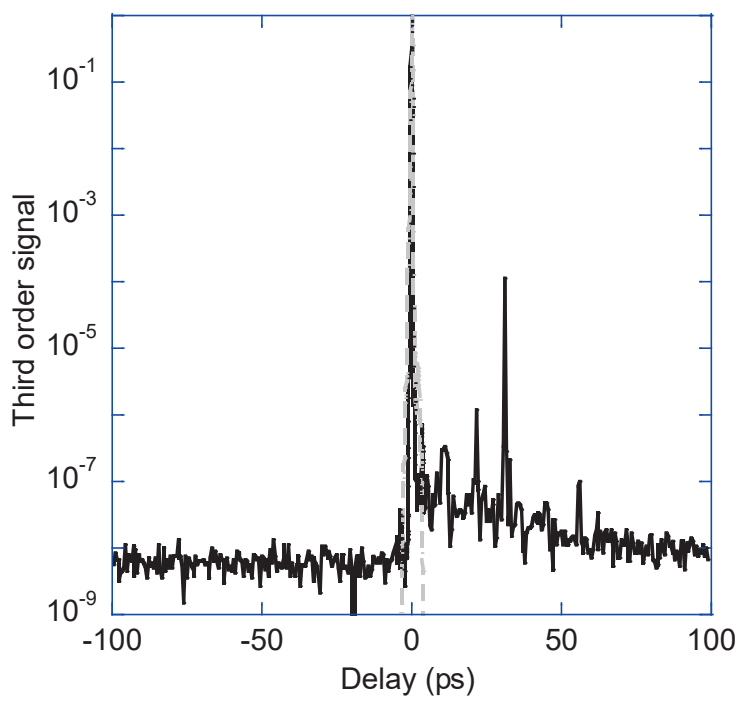

Fig. 2 Measured third order correlation after the pulse cleaner (solid line). Calculated $I_{\text {signal }}(t)^{3} \quad$ (dashed line).

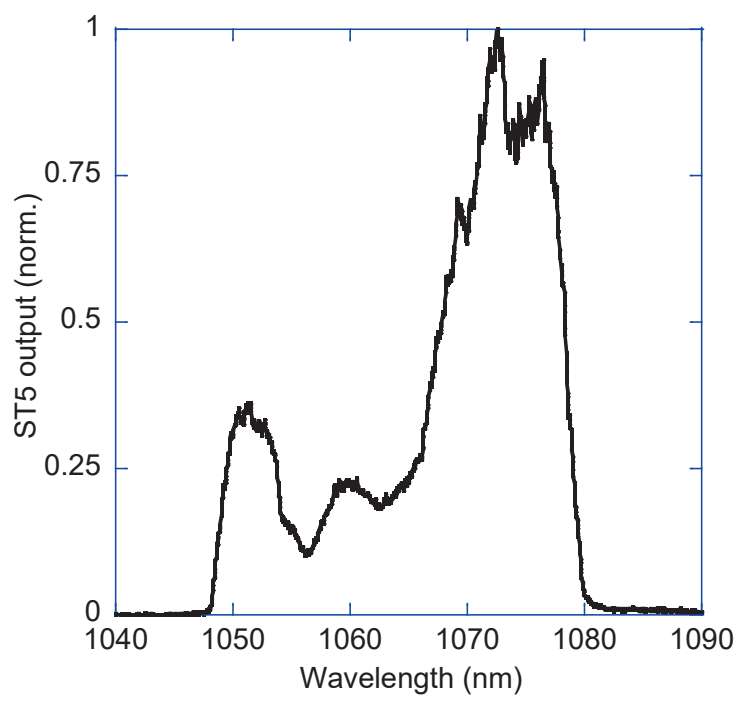

Fig. 3 Spectrum at the output of the FE.

ian to square flat-top by a serrated aperture, and the shape is maintained by between OPCPA stages. The spatial flat-top is flattened on the edges by saturation through the ns OPCPA. Figure 4 shows spatial profile after stage 5 .

\section{Power Amplifiers}

Amplification of laser pulses in glass media has already been demonstrated up to $\sim 20 \mathrm{~kJ}$ for narrow linewidth nanosecond pulses and up to 150 Joules for large spectral bandwidth pulse (150 fs). ${ }^{13)}$ The Power Amplifiers (PA) section aims to bring the energy up to 1700 Joules on a large bandwidth compatible with 130 fs FTL duration while allowing one shot a minute repetition rate. To achieve these 2 specifications, this section is composed of two amplifiers (PA1 and PA2) relying on the mixed-glass liquid cooled split-disk technology. Each amplifier consists of a full reflective relay imaging system allowing four passes in the amplification modules. Silicate and phosphate glass are used for bandwidth management and high energy amplification. Phosphate has a peak gain at $1052 \mathrm{~nm}$ while the peak gain of silicate is at $1060 \mathrm{~nm}$. When these two glasses types are used in an appropriate ratio in amplifiers, the result is a broad gain bandwidth while keep-

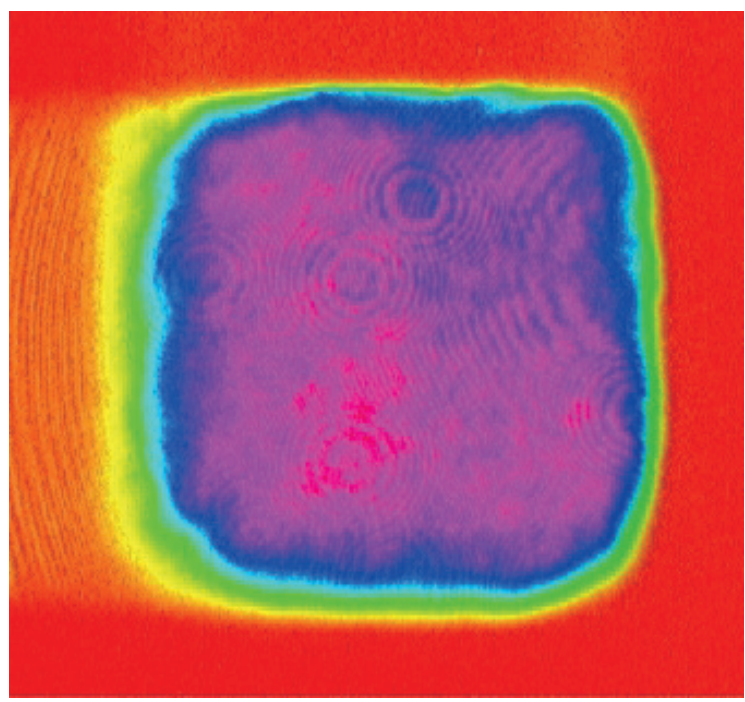

Fig. 44 Joules, $5 \mathrm{~Hz}$, output spatial beam profile of FE. 
ing a high amplified energy.

Despite this enlarged bandwidth and as mentioned in paragraph 2, the spectral gain narrowing will inevitably reduce width of the final output spectrum.

Strong shaping of the input spectrum is therefore required. The experimental OPCPA output spectrum (Fig. 3) seeding the PA section, allows a $10 \mathrm{PW}$ pulse at the output. Figure 5 shows simulated amplified spectra with an energy of 1700 Joules.

The thermal management to allow operation at one shot a minute is obtained by using a split-disk arrangement with liquid coolant in between glass slabs. Figure 6 shows a PA1 module assembly consisting of a cartridge on both sides with flash-lamps (water cooled) and the split-disk glass slabs which are actively liquid-cooled by Fluorinert. A deformable mirror is implemented in each power amplifier for wavefront optimi-

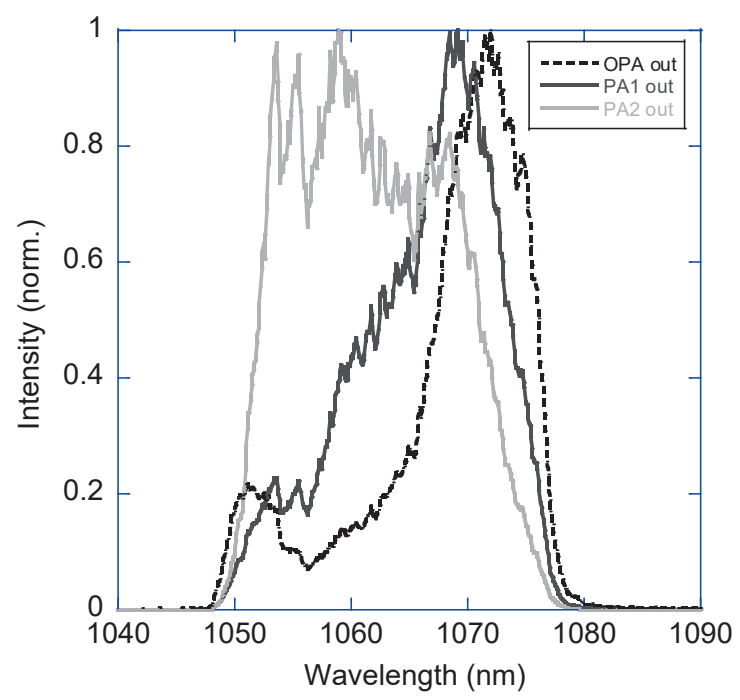

Fig. 5 Calculated spectra after PA1 and PA2 (respectively black line, gray line). The input spectrum from the OPCPA section, dotted line, is measured experimentally.

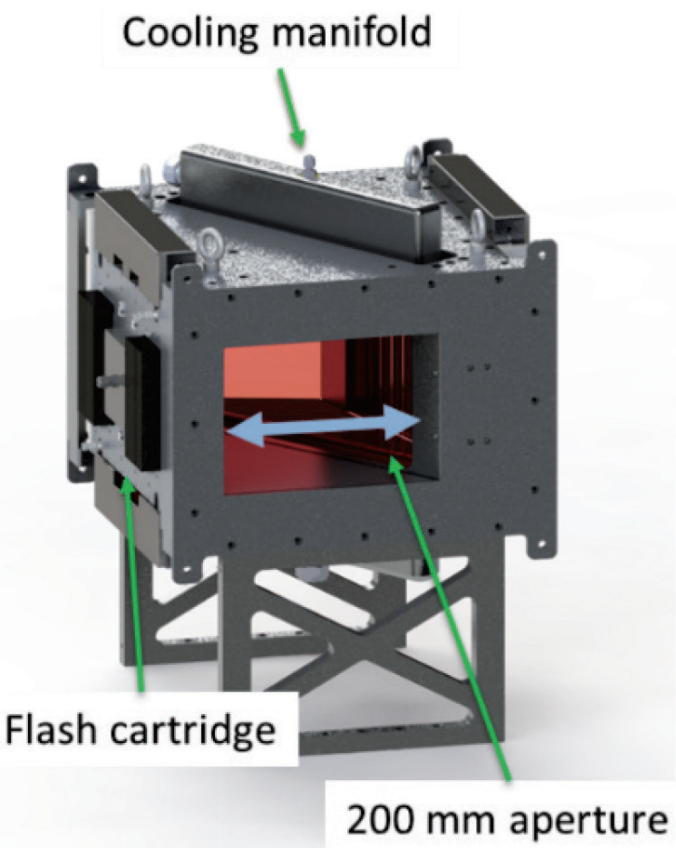

Fig. 6 Amplifier module used in the first power amplifier. zation.

The first amplifier is fully assembled, and has first been characterized with no amplification. Figure 7 shows the PA1 output focal spot. The associated Strehl ratio is 0.75 .

We are currently ramping up the amplified energy. Up to 2.35 Joules pulses with shaped spectrum has been seeding the amplifier. Amplified energy is 38.1 Joules. Figure 8 shows results in terms of output beam profile and spectrum.

We expect then a pulse energy of 65 Joules when this amplifier will be seeded by the 4 Joules from the OPCPA section.

This stage can produce $1 \mathrm{PW}$ pulses under a different seed spectrum, thanks to the OPCPA shaping capabilities. The spectrum will therefore be centered at $1060 \mathrm{~nm}$ and pulse will experience much more gain. Figure 9 is showing results of the simulation.

The second power amplifier has the same structure with modules containing the liquid-cooled phosphate slabs and a four-pass configuration with a beam size of $26 \mathrm{~cm}$. It is currently under construction.

\section{Compression}

The amplified beam will be transported to the optical compressor through the Compressor Imaging System or CIS. Reflective optics are used for relay imaging and up collimating the square beam to $620 \mathrm{~mm}$. A deformable mirror will be implemented within the CIS to ensure a flat wavefront on the first grating for optimal pulse compression. The optical compressor is made from four gratings in an $\mathrm{X}$ configuration (Fig. 10). They have 1136 lines/mm and are dielectric coated with a diffraction efficiency over $98 \%$. They will be under Littrow angle while out of the diffraction plane. The distance

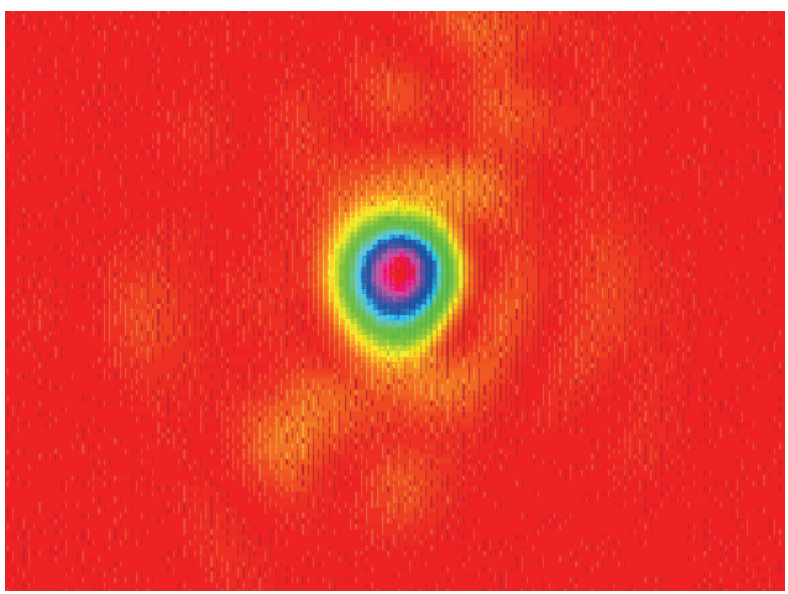

Fig. 7 Measured focal spot at the output of Power Amplifier 1 (unamplified).
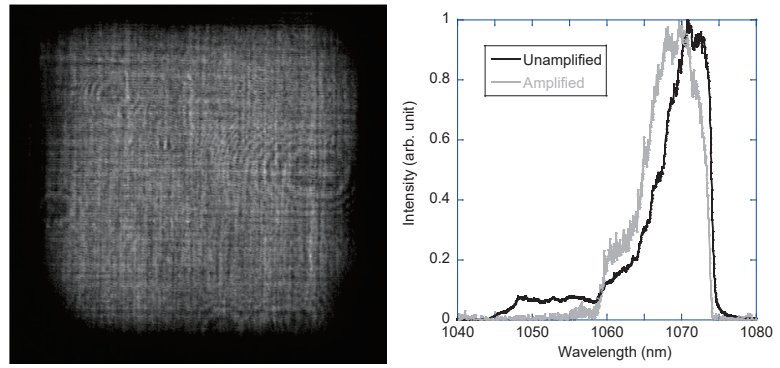

Fig. 838 Joules output beam profile of PA1 (left). Input and output spectrum (right). 


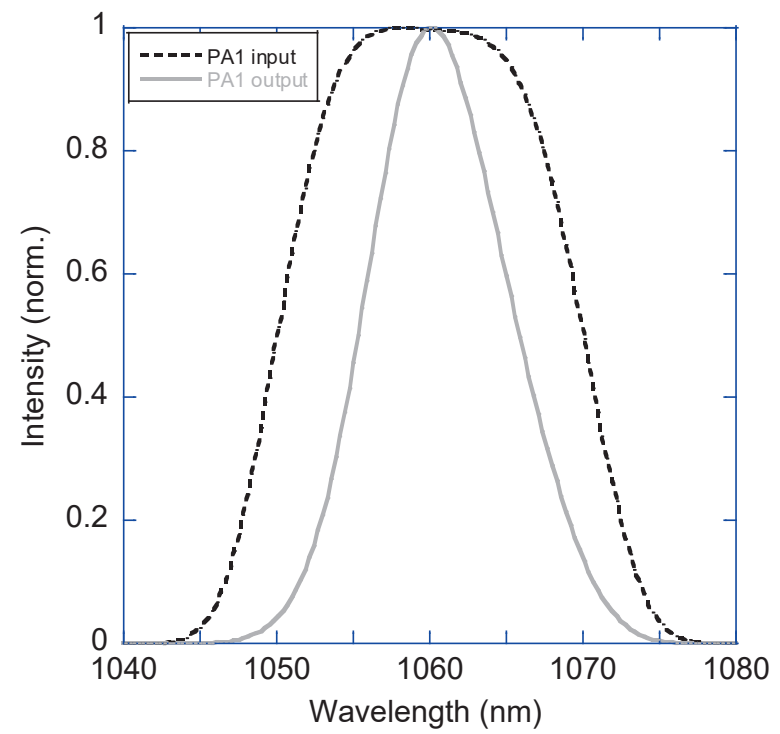

Fig. 9 Input spectrum (dotted line) and output spectrum (solid line) for $1 \mathrm{PW}$ operation.

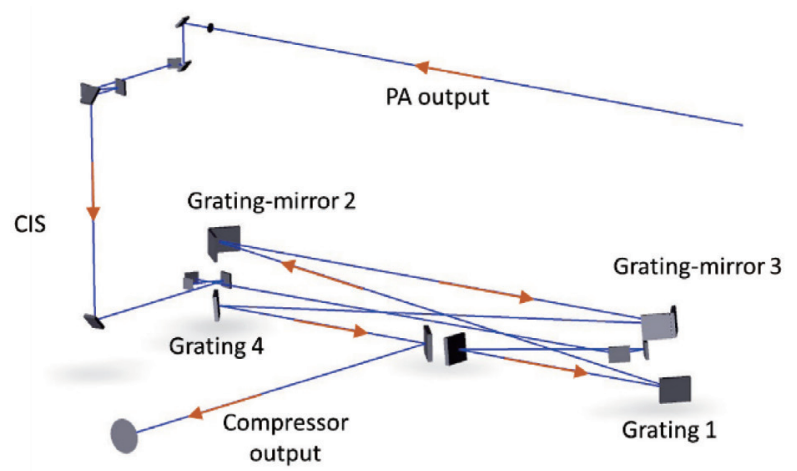

Fig. 10 Optical configuration of the Compressor Imaging System and optical compressor.

between gratings is 15 meters.

The beam dimension on gratings 3 and 4 is more than 1 meter. Monolithic gratings of the required size do not exist, so alternative methods must be implemented. One option is phasing smaller gratings.

The approach we have selected to solve this problem is to phase the grating with a mirror at $90^{\circ}{ }^{17)}$ This solution is easier to implement as the line-density variation, angular tip, and longitudinal piston errors within a tiled grating no longer exist. The number of degrees of freedom to consider are then lowered. The CIS and optical compressor will be implemented in a large vacuum vessel.

\section{Conclusion}

The $10 \mathrm{PW}$ laser under construction by National Energetics is planned to be commissioned by next spring.

There are multiple approaches toward $10 \mathrm{PW}$ which are currently being pursued at research facilities around the world. One of the most common routes is conventional Ti:Sapphire amplification of ultra-short pulses (generally -20 fs pulse duration). The path toward this goal taken by the ELI-Beamlines $10 \mathrm{PW}$ beamline, under development by a consortium of National Energetics and Ekspla with support from ELI-Beamlines staff, is to amplify longer pulses (approx. $100 \mathrm{fs}$ ) to $\mathrm{kJ}$-level energies. This pushes Nd:glass amplification further than ever in terms of broadband output energy at an unprecedented repetition rate of 1 shot per minute. The $4 \mathrm{~J}, 5 \mathrm{~Hz}$ OPCPA-based front end has proven to be a suitable seed pulse for the $10 \mathrm{PW}$ beamline in terms of customizable spectrum, contrast, and spatial profile. The first results obtained on PA1 (overall gain and amplified spectrum) show output values compatible with the required inputs on PA2 for a $10 \mathrm{PW}$ operation. When commissioned, this merger of glass amplification and broadband OPCPA will provide a $\mathrm{kJ}$ class femtosecond laser system to the user community and unique opportunities for study in high field physics.

\section{References}

1) J. Hee Sung, H. W. Lee, J. Y. Yoo, J. W. Yoon, C. W. Lee, J. M. Yang, Y. J. Son, Y. H. Jang, S. K. Lee, and C. H. Nam: Opt Lett. 42 (2017) 2058.

2) D. Papadopoulos, J. Zou, C. Le Blanc, G. Chériaux, P. Georges, F. Druon, G. Mennerat, P. Ramirez, L. Martin, A. Fréneaux, et al.: High Power Laser Sci. Eng. 4 (2016) e34.

3) Y. Chu, Z. Gan, X. Liang, L. Yu, X. Lu, C. Wang, X. Wang, L. Xu, H. Lu, D. Yin, et al.: Opt Lett. 40 (2015) 5011.

4) X. Zeng, K. Zhou, Y. Zuo, Q. Zhu, J. Su, X. Wang, X. Wang, X. Huang, X. Jiang, D. Jiang, et al.: Opt Lett. 42 (2017) 2014.

5) V. V. Lozhkarev, G. I. Freidman, V. N. Ginzburg, E. V. Katin, E. A. Khazanov, A. V. Kirsanov, G. A. Luchinin, A. N. Mal'shakov, M. A. Martyanov, O. V. Palashov, et al.: Laser Phys. Lett. 4 (2007) 421.

6) X. Zeng, K. Zhou, Y. Zuo, Q. Zhu, J. Su, X. Wang, X. Wang, X. Huang, X. Jiang, D. Jiang, et al.: Opt Lett. 42 (2015) 2014.

7) G. Cerullo and S. De Silvestri: Rev. Sci. Instrum. 74 (2003) 1.

8) C. A. Haynam, P. J. Wegner, J. M. Auerbach, M. W. Bowers, S. N. Dixit, G. V. Erbert, G. M. Heestand, M. A. Henesian, M. R. Hermann, K. S. Jancaitis, et al:: Appl. Opt. 46 (2007) 3276.

9) J.-L. Miquel, C. Lion, and P. Vivini: J. Phys.: Conf. Ser. 688 (2016) 012067.

10) N. Blanchot, C. Rouyer, C. Sauteret, and A. Migus: Opt Lett. 20 (1995) 395.

11) M. D. Perry, D. Pennington, B. C. Stuart, G. Tietbohl, J. A. Britten, C. Brown, S. Herman, B. Golick, M. Kartz, J. Miller, et al.: Opt Lett. 24 (1999) 160.

12) C. Dorrer, A. Consentino, D. Irwin, J. Qiao, and J. D. Zuegel: J. Opt. 17 (2015) 094007

13) E. Gaul, M. Martinez, J. Blakeney, A. Jochmann, M. Ringuette, D. Hammond, T. Borger, R. Escamilla, S. Douglas, W. Henderson, et al.: Appl. Opt. 49 (2010) 1676.

14) Y. J. Wang and B. Lutherdavies: J. Opt. Soc. Am. B 11 (1994) 1531.

15) R. C. Shah, R. P. Johnson, T. Shimada, K. A. Flippo, J. C. Fernandez, and B. M. Hegelich: Opt Lett. 34 (2009) 2273.

16) G. Cheriaux, P. Rousseau, F. Salin, J. P. Chambaret, B. Walker, and L. F. Dimauro: Opt. Lett. 21 (1996) 414.

17) Z. Li, G. Xu, T. Wang, and Y. Da: Opt Lett. 35 (2010). 